Archives de sciences sociales des religions

$177 \mid 2017$

Mondes juifs en mouvement

\title{
Lumières sur la ville
}

Les fêtes de Hanoucca entre action missionnaire transnationale et appartenance événementielle

Lucine Endelstein

\section{(2) OpenEdition}

Journals

Édition électronique

URL : http://journals.openedition.org/assr/29252

DOI : $10.4000 /$ assr.29252

ISSN : $1777-5825$

Éditeur

Éditions de l'EHESS

Édition imprimée

Date de publication : 1 mars 2017

Pagination : 51-71

ISSN : 0335-5985

\section{Référence électronique}

Lucine Endelstein, «Lumières sur la ville », Archives de sciences sociales des religions [En ligne], 177 | 2017, mis en ligne le 01 mars 2019, consulté le 06 janvier 2021. URL : http://journals.openedition.org/ assr/29252 ; DOl : https://doi.org/10.4000/assr.29252 


\section{Lucine Endelstein}

\section{Lumières sur la ville}

\section{Les fêtes de Hanoucca entre action missionnaire transnationale et appartenance événementielle}

Des places centrales et symboliques des grandes métropoles jusqu'aux confins des agglomérations, de Buenos Aires à Sidney en passant par New York, Munich et New Delhi, la fête de Hanoucca est aujourd'hui célébrée dans les espaces publics urbains à travers le monde entier. Cette fête transnationale témoigne de façon spectaculaire du succès du mouvement hassidique Loubavitch ${ }^{1}$ qui l'organise depuis la fin des années 1970 (Gutwirth, 2004). C'est surtout depuis les années 2000 que ces fêtes se sont multipliées et participent d'une mise en spectacle recherchée par le mouvement Loubavitch pour diffuser ses messages dans une perspective missionnaire, interne au monde juif, qui vise à ramener un maximum de juifs à l'accomplissement des commandements divins. Les allumages publics des bougies de Hanoucca s'inscrivent dans la série des marches, parades et autres performances publiques menées par d'autres religions (Abdullah, 2009; Baby-Collin et Sassone, 2010 ; Fer, 2007 ; Garbin, 2012b), associées à la notion de « religions urbaines »(Orsi, 1999) et qui prennent l'espace urbain comme terre de mission.

La littérature récente portant sur les phénomènes contemporains de pratique religieuse événementielle, articulant fortement le local au global, a montré que ces derniers sont des expressions d'appartenance collective (Salzbrunn, 2014). De plus, comme le fait remarquer David Garbin, «l'expérience religieuse urbaine des groupes diasporiques - à travers leur visibilité et leur oralité, promulgue la différence, en nous faisant réfléchir à la manière dont les frontières entre insiders et outsiders, public et privé, sacré et profane, peuvent jouer un rôle crucial dans les politiques de l'appartenance et des identités ${ }^{2}$ » (Garbin, 2012a). Les événements religieux en public marquent ainsi un mode

1. Le mouvement Loubavitch, également appelé Habad, est un mouvement hassidique né en Europe orientale au XVIII ${ }^{\mathrm{e}}$ siècle. Chacune des branches hassidiques est organisée autour d'un leader spirituel descendant d'une dynastie, et porte le nom de la ville ou du village où elle est apparue. Voir (Baumgarten, 2005). Sur le développement du mouvement Loubavitch en France voir (Podselver, 2010).

2. Traduit de l'anglais. 
d'appartenance dans un collectif local et diasporique, et s'inscrivent avec d'autres processions, marches et carnavals dans la recherche d'un droit moral à exister.

Au-delà du sens religieux et missionnaire qui leur est attribué par le mouvement Loubavitch, les fêtes de Hanoucca offrent des occasions privilégiées pour examiner le rôle de la religion sous sa forme transnationale, événementielle et spectaculaire, dans la construction et la reformulation du sentiment d'appartenance au monde juif et dans la mise en scène d'une différence qui ouvre un échange avec la société urbaine. Quels sont alors les enjeux de la pérennisation de ces festivités pour la construction de l'image du monde juif exposé en public?

Pour répondre à ces interrogations les analyses présentées ici reposent sur des observations de ces festivités et sur des enquêtes par questionnaires courts menées in situ auprès du public sur plusieurs places de Paris et de Londres ${ }^{3}$. Peu d'attention a été portée jusqu'à présent à la diversité interne du public qui s'expose pendant les événements religieux spectaculaires tels que les fêtes, parades et processions (Abdullah, 2009; Eade et Garbin, 2007). Pourtant donner des voix aux publics de ces événements permet de mettre en lumière les clivages et la porosité des frontières d'un groupe souvent perçu comme harmonieux et "fermé ». Les témoignages recueillis montrent comment se résout dans l'émotion d'un rassemblement éphémère l'opposition entre la diversité du groupe et l'unité de sa représentation, à laquelle fait écho sa désignation courante comme une "communauté».

Mener des enquêtes dans des contextes urbains et nationaux différents permet de saisir l'articulation d'échelles à l'œuvre dans les pratiques religieuses transnationales, qui combinent à la fois des processus d'homogénéisation et de différenciation (Capone, 2010). Ces derniers tiennent au succès d'un mouvement religieux planétaire qui tend à homogénéiser des pratiques urbaines, aux histoires de l'insertion géographique des juifs dans les deux capitales, ainsi qu'aux différences du statut public de la religion dans deux grandes démocraties européennes aux modèles de société souvent présentés comme opposés. Avec ces variations locales comme trame de fond, différents processus communs sont ici exposés: tout d'abord, la dimension missionnaire de la fête pour le mouvement Loubavitch et ses stratégies d'ouverture sociale et politique; ensuite, les sens multiples de la fête pour le public juif qui dessine un continuum depuis les Loubavitch les plus militants jusqu'aux juifs éloignés du monde juif et de ses traditions; enfin la rencontre entre le monde juif et la société à l'occasion de ces fêtes et les enjeux de leur pérennisation.

3. À Paris en 2011, place du Trocadéro, place de l'Opéra, place de l'Étoile, place de la Bastille et place des Fêtes; à Londres en 2015 (Trafalgar Square, Golders Green). Les principales questions traitées dans le cadre de cet article portent sur les raisons de la présence à l'événement et sur le sens donné à la fête, ainsi que sur la pratique et l'auto-identification religieuse. 


\section{Hanoucca, une fête transnationale et missionnaire}

C'est à New York, en 1977 que le rav Menachem Mendel Schneerson, chef spirituel du mouvement Hassidique Habad, a lancé ces allumages publics en leur attribuant la mission de soutenir la «survivance spirituelle de la flamme juive ». Le premier allumage à Paris a eu lieu sur la place des Fêtes, près du premier centre Habad parisien, en 1980. Ces célébrations se sont progressivement étendues aux grandes métropoles du monde entier, et dans tous les lieux où les shluchim, les émissaires du rabbi, sont installés pour diffuser et promouvoir le judaïsme et le hassidisme dans une perspective missionnaire et messianique, l'attente de l'arrivée imminente du Messie étant une caractéristique importante du mouvement ${ }^{4}$.

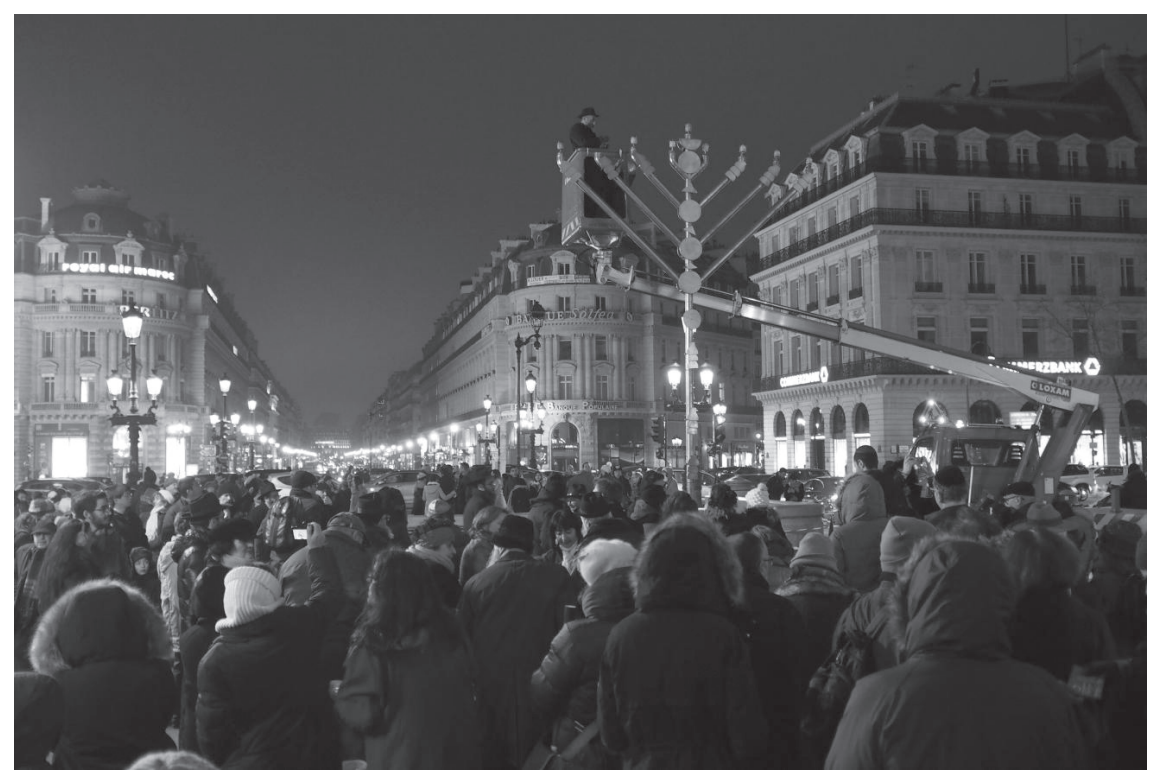

Photo 1. Allumage du candélabre place de l'Opéra, décembre 2016 @ Guillaume Poux

Le message de la fête de Hanoucca est particulièrement adapté à cette perspective missionnaire. Cette fête rabbinique commémore en effet la réinauguration du second Temple de Jérusalem au $\mathrm{II}^{\mathrm{e}}$ siècle avant Jésus-Christ, suite à la victoire des Maccabées sur les Grecs et les troupes d'Antiochus IV. L'allumage de bougies pendant les huit jours de la fête symbolise le miracle qui, selon la tradition rabbinique, s'est produit lors de la réinstallation du culte dans le Temple: le fait qu'une très petite quantité d'huile a suffi pour

4. Le mouvement Loubavitch a développé à partir des années 1980 un très fort messianisme qui s'est tourné vers son leader, le Rabbi Menahem Mendel Schneerson. Sa mort en 1994 provoqua une profonde division au sein du mouvement. Voir Kravel-Tovi (2009). 
brûler pendant huit jours. La lumière de cette fête d'hiver représente aussi la résistance spirituelle juive dans un milieu voué à la faire disparaître. Cette fête est très suivie aujourd'hui dans le monde juif même par les familles qui se définissent comme laïques: joyeuse, peu contraignante car il ne s'agit pas de jours chômés, Hanoucca qui tombe pendant la période de Noël est devenue la fête des enfants et l'occasion de s'offrir des cadeaux.

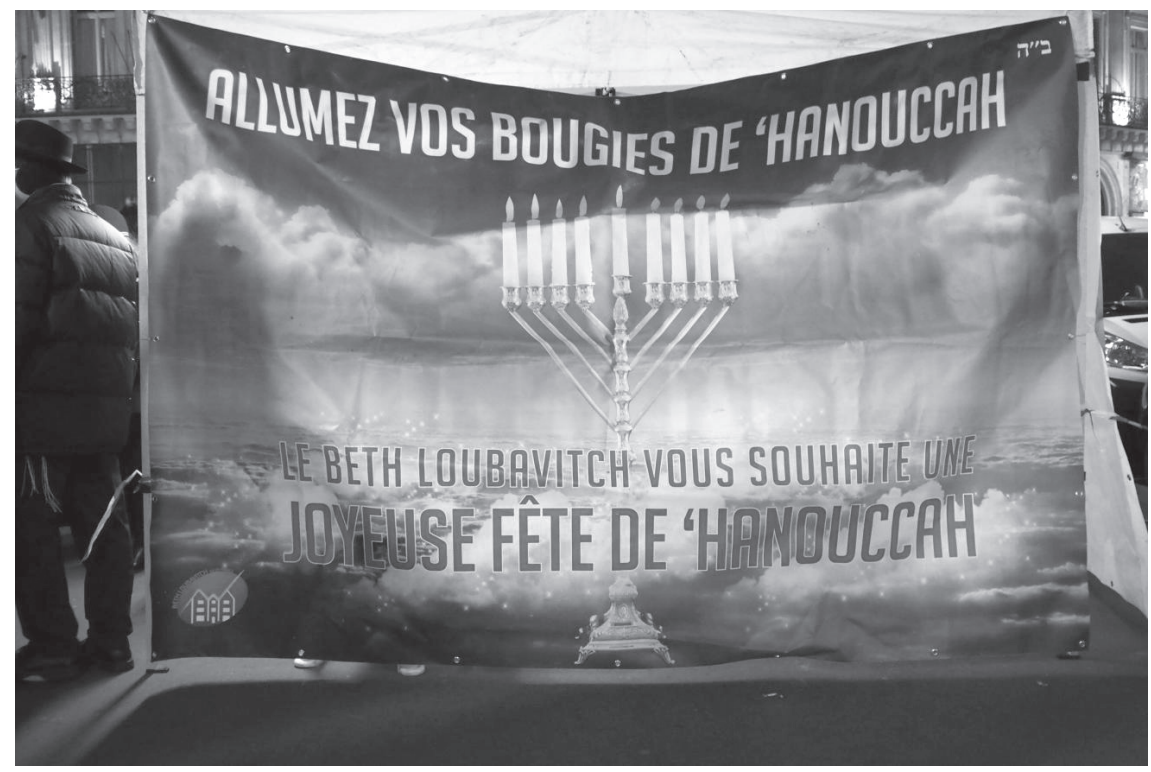

Photo 2. Message affiché sur un stand par le mouvement Loubavitch, place de l'Opéra, décembre 2016 @C Guillaume Poux

Quel que soit le lieu, le déroulement des festivités est quasiment identique et dure environ une heure. Trois objets symbolisant la fête et l'action du mouvement Loubavitch attribuent une fonction festive à l'espace. Tout d'abord un candélabre géant, qui sera le centre de l'attention, est dressé sur le lieu de la fête juste avant qu'elle ne commence (photo 1). Il sera rapidement démonté immédiatement après la célébration. Ensuite une ou plusieurs tentes sont placées à proximité, abritant la régie musicale ou un orchestre, ainsi qu'un stand de distribution de différents objets: prospectus expliquant le sens de la fête et détaillant le procédé de l'allumage des bougies, candélabres jetables, bougies et beignets distribués gracieusement. Le message adressé à la foule par le mouvement Loubavich est inscrit en grands caractères sur cette tente: "Allumez vos bougies de Hanoucca. Le Beth Loubavitch vous souhaite une joyeuse fête de Hanoucca» (photo 2). Enfin, l'arrivée bruyante d'une voiture diffusant une musique hassidique, et sur le toit de laquelle est fixée une hanoukkia lumineuse, annonce le début des festivités. La fête débute ainsi par la diffusion de musiques hassidiques et klezmer, qui en donnent le ton joyeux. 
Au cœur de l'événement, des représentants du mouvement Loubavitch, des responsables communautaires et parfois des personnalités du monde politique ou de la culture se succèdent sur une grue qui les hisse à la hauteur du candélabre. Ils y prononcent des discours puis allument les torches électriques qui symbolisent les bougies.

Le rituel religieux est accompli publiquement par la brève énonciation de bénédictions en hébreu au moment de l'allumage des lampes, à laquelle la foule répond en chœur "Amen». Cet instant solennel ritualise le particularisme du rassemblement juif, mais il est intégré dans une ambiance festive et ouverte sur la ville où tout passant est convié. Si les frontières externes du monde juif s'effacent, les frontières de genre établies par les mouvements ultra-orthodoxes sont strictement respectées et mises en scène lors de ces festivités: les hommes en costume sombre, barbe et chapeaux du mouvement Loubavitch ne tardent pas à effectuer des danses traditionnelles, s'enlaçant par les épaules et tournant en cercle. Leurs femmes restent spectatrices car la tsiniout - ou règles de la pudeur - leur interdit de danser sous le regard des hommes. Il arrive de voir d'autres femmes danser, mais ces dernières ne font pas partie du mouvement Loubavitch ou d'autres courants ultra-orthodoxes. Musique, lumières, danse, spectacle: l'ambiance de cette fête qui se déroule au mois de décembre se fond dans l'ambiance de Noël et dans les lumières qui illuminent la ville au creux de l'hiver.

\section{Pratiques transnationales, variations locales}

Déroulement identique, objets similaires et diffusés dans le monde entier par le même mouvement religieux, similitude des messages qui circulent: cette fête donne à voir des processus d'homogénéisation propres à la globalisation et aux pratiques religieuses transnationales (Capone, 2010). Mais même s'ils portent la marque du global, ces événements ne sont pas rigoureusement similaires d'un lieu à l'autre et encore moins « déterritorialisés ». Au contraire chacun met en scène à la fois des pratiques religieuses transnationales et les particularités d'un contexte local et national, et c'est cette articulation d'échelles qui se donne à lire chaque soir et en chaque lieu.

Les lieux de la fête reflètent dans chaque pays récepteur de ces pratiques religieuses transnationales les variations de la place du monde juif dans sa société d'installation. Il est révélateur par exemple qu'à Madrid une seule soirée d'allumage public soit organisée chaque année, sur une place de taille modeste et non sur un lieu emblématique de la Nation, ce qui reflète à la fois la faible importance démographique du monde juif espagnol et la tardive liberté religieuse promulguée dans un pays longtemps marqué par la dictature. À Paris et à Londres, capitales de démocraties anciennes où la liberté religieuse est inscrite de longue date dans les textes fondamentaux, et capitales du monde juif européen, les allumages ont lieu tous les soirs et sur des places centrales. À Paris, de très nombreux allumages sont organisés, des places les plus symboliques (place de la République, place de l'Opéra, place de la Bastille, place 
de l'Étoile, etc.) jusqu'aux places périphériques des villes de banlieue, mettant en lumière la diffusion du mouvement Loubavitch dans toute l'agglomération parisienne. À Londres, les allumages sont resserrés sur une seule place centrale, Trafalgar Square, ainsi que dans quelques quartiers marqués par une forte présence juive orthodoxe et ultra-orthodoxe, notamment Golders Green et Stamford Hill. Ces variations reflètent les différences de l'insertion urbaine des juifs dans les deux capitales: alors qu'il existe à Londres d'importantes centralités juives orthodoxes, la géographie du judaïsme dans l'agglomération parisienne est marquée par une plus grande dissémination.

Une large littérature a montré l'inversion de l'ordre social vécu dans l'expérience éphémère des fêtes et carnavals. Dans le cas de ces groupes religieux minoritaires qui prennent place sur les lieux symboliques des sociétés d'installation, il s'agit aussi d'une inversion de l'ordre spatial. En effet les lieux de culte du judaïsme sont historiquement situés dans des rues périphériques et non pas des places centrales, souvent intégrées dans la perspective des rues comme les synagogues consistoriales parisiennes (Jarrassé, 2001), ou dissimulées dans des impasses comme dans l'East End de Londres (Kershen et Vaughan, 2013). Les synagogues bâties à l'époque contemporaine se caractérisent toujours par une relative discrétion, contrastant avec la spectacularisation des fêtes de Hanoucca qui donne une occasion de sortir des lieux périphériques pour prendre place en pleine lumière au cœur du monde urbain.

Les déroulements des fêtes à Paris et à Londres portent l'empreinte des deux politiques de la diversité religieuse française et britannique. Organiser une fête religieuse sur la plus grande place de Londres est conforme à la conception multiculturaliste de la nation où les Faith communities sont institutionnalisées comme interlocuteurs avec l'État (Beckford, 2015). C'est une mise en scène de ce dialogue entre les autorités publiques et des collectifs politiquement définis comme des "communautés » religieuses - considérées sans doute à tort comme des communautés de foi (ibid.) - qui a lieu à Trafalgar Square où le Maire de Londres ne rate aucun de ces rendez-vous annuels avec «la communauté juive». À Paris les autorités publiques ne se rendent pas en personne sur le lieu d'allumage majeur, celui du Champ de Mars, mais transmettent leurs vœux. Malgré les différences politiques qui opposent le multiculturalisme britannique et la laïcité française qui reconnaît l'individu-citoyen avant les groupes religieux, on voit qu'il existe au cours de l'événement festif un acte de reconnaissance d'une minorité religieuse, différemment mis en scène.

Enfin la résonnance mondiale de la fête de Hanoucca est mise en scène à Paris lors du rassemblement du Champ de Mars. Des écrans géants conjuguent en duplex ces célébrations avec celles de New York et de Jérusalem, et matérialisent le fort ancrage local de pratiques religieuses transnationales (Levitt, 2007). Avec les nouvelles technologies de l'information et de la communication les modalités de célébration de la fête publique de Hanoucca transcendent les frontières étatiques, rassemblant virtuellement ces trois lieux phares de la diaspora juive. Avec cette mise en spectacle de la diaspora et de ses liens avec 
Israël, le transnational s'ancre dans le local pour réactualiser le sentiment d'appartenance à un collectif dispersé dans l'espace et dans le temps.

L'utilisation de l'image et d'une technologie de pointe peut surprendre de la part d'un mouvement juif ultra-orthodoxe, qui perçoit la société moderne comme une source de perdition, et souvent perçu lui-même comme rétrograde. Cet usage rappelle pourtant que la technologie est aujourd'hui un vecteur prodigieux de diffusion des messages religieux y compris les plus radicaux (Campbell, 2013; Duteil-Otaga et al., 2015) et que les mouvements radicaux accompagnent le processus de modernisation des sociétés jusqu'à être considérés par certains auteurs comme un "symptôme» de cette modernisation (Fath, 2010).

Cette diffusion des fêtes des places centrales jusqu'aux lieux plus périphériques des métropoles parisienne et londonienne exprime dans l'espace urbain l'action missionnaire et la stratégie d'ouverture qui font la particularité du mouvement Loubavitch au sein du monde hassidique (Gutwirth, 2004).

\section{Action missionnaire et stratégies d'ouverture}

Placer des candélabres géants sur des places publiques, au cœur de la cité aujourd'hui, vise à envoyer des messages aussi bien aux juifs les plus pratiquants qu'aux plus éloignés de la religion. En démultipliant les allumages et en incitant un maximum de juifs à procéder à la mitsva de l'allumage des bougies chez eux, les membres du mouvement espèrent hâter la venue du Messie: "Ainsi, très bientôt viendra le temps où "nous allumerons les lumières dans ton sanctuaire ", par la venue du Machia’h» écrit le rav Schneerson en $1978^{5}$. «Illuminer le monde» c'est ainsi pour le mouvement Loubavitch s'adresser à la société en affirmant la victoire de la lumière de la liberté sur la «nuit de l'oppression ${ }^{6}$ ".

Mais ces événements polysémiques ne s'adressent pas uniquement au monde juif, et les messages émis lors des allumages des bougies sont souvent explicitement dirigés vers la société dans son ensemble, comme le discours prononcé place de l'Étoile par le rabbin Haïm Nissenbaum, leader du mouvement Loubavitch à Paris: "Que vous soyez israélite ou d'autres religions, vous êtes les bienvenus. Hanoucca se fête dans la rue, car le message de Hanoucca est universel, pour toute l'Humanité ». Cet usage de la rue comme scène de diffusion de messages rappelle que les lieux où ces fêtes prennent place, en tant qu'espaces publics ou endroits accessibles au(x) public(s), arpentés par les habitants, remplissent une fonction essentielle de la vie collective et de l' "urbanité élémentaire" (Paquot, 2015): la communication. La rencontre et l'échange, la mise en scène d'une différence lors de fêtes comme celles de Hanoucca contribuent à unir les «espaces publics matériels de la circulation» et l' "espace public métaphorique de la communication» (ibid., 2015). La terminologie goffmanienne du «théâtre

5. http://www.fr.chabad.org/library/article_cdo/aid/601900/jewish/La-lumire.htm

6. http://www.fr.chabad.org/library/article_cdo/aid/594687/jewish/Illuminons-le-monde.htm 
urbain », considérant que la ville toute entière est espace de représentation, est parfaitement adaptée pour ces fêtes initialement religieuses mais qui interviennent dans un processus séculier: celui par lequel un groupe "prend place» (Joseph, 1995) dans la ville et met en scène son particularisme lors de spectacles joués par et pour les citadins.

Lors de ces festivités urbaines et dans tous les contextes nationaux, les Loubavitch mènent une double stratégie d'ouverture: tout d'abord une ouverture "vers le haut», en établissant des liens avec les autorités municipales et préfectorales pour l'organisation de leurs manifestations publiques. Or s'engager dans l'organisation d'événements apporte une caution aux acteurs qui échangent avec les pouvoirs locaux (Di Méo, 2005). Le fait que le mouvement hassidique Loubavitch se positionne lors de ces fêtes publiques comme le représentant de "la communauté » juive et comme son interlocuteur auprès des autorités publiques locales pose la question de l'enjeu politique des célébrations et de la légitimation d'un mouvement ultra-orthodoxe, rivalisant ainsi avec les institutions historiquement établies pour représenter le judaïsme (Consistoire en France, United Synagogues au Royaume-Uni).

Le mouvement travaille également à l'établissement de bonnes relations avec l'ensemble du monde juif institutionnel, en conviant à prononcer des discours de nombreux responsables et personnalités. Ensuite, la stratégie d'ouverture «vers le bas » consiste à mener une diffusion sociétale en se présentant comme les gardiens des traditions juives, en cherchant tout aussi bien à renforcer et «orthodoxiser» la pratique religieuse des juifs déjà pratiquants mais sur un mode plus traditionnel, et à ramener des juifs non pratiquants vers la techouva, c'est-à-dire vers la pratique du judaïsme ${ }^{7}$. La visibilité du mouvement Loubavitch dans l'organisation des célébrations fait partie de sa recherche d'influence au sein du monde juif. Le militantisme Loubavitch contribue ainsi à des changements au sein de la population juive, tout en exerçant à la fois une concurrence et une influence sur les institutions juives dans les deux contextes.

\section{Sens multiples de la fête}

\section{« Illuminer le monde»}

Pour les membres les plus actifs du mouvement Loubavitch, venir allumer publiquement les bougies de Hanoucca représente tout d'abord l'accomplissement d'une mitsva ${ }^{8}$ : "Je suis ici parce que je suis juive et je dois allumer les bougies », dit à Golders Green une jeune femme ${ }^{9}$ qui se déclare orthodoxe et

\footnotetext{
7. Si cette stratégie a bien fonctionné au cours des dernières décennies dans le monde, le phénomène de techouva en France est original au sens où il concerne en grande partie - mais pas exclusivement - un public sépharade qui prend certaines distances avec les traditions familiales originaires d'Afrique du Nord pour adopter des pratiques hassidiques venant d'Europe orientale. 8. Une mitsva est une prescription contenue dans la Torah (elles sont traditionnellement estimées à 613) ou dans la Loi juive dans son ensemble.

9. Femme née en 1981, puéricultrice.
} 
fréquentant une synagogue Habad. Pour les Loubavitch le sens religieux de la fête est donné spontanément comme la première raison de la participation à l'événement: "(Je participe à cet événement) pour commémorer ce grand miracle qui a eu lieu il y a deux mille ans, quand ils ont voulu détruire le peuple Juif » déclare un jeune homme ${ }^{10}$. Le mouvement Loubavitch étant organisé autour de la personnalité du « rabbi » Schneerson, l'accomplissement de sa volonté est avancée comme l'une des raisons de l'organisation de la fête, comme poursuit ce jeune homme Loubavitch londonien: "Le rabbin a dit que nous devions organiser les allumages en public. Plus vous faites cela en public, plus vous rapprochez les gens du judaïsme». Dans cette perspective ouvertement missionnaire, les lumières de Hanoucca s'adressent à tous les juifs afin de les ramener vers l'accomplissement des mitsvot ${ }^{11}$.

Nombre de participants appartenant au mouvement Loubavitch insistent sur l'universalité de la dimension spirituelle de la fête, qui doit franchir les frontières du monde juif. Place de la Bastille, une femme très active dans le mouvement Loubavitch à Paris et qui suit tous les allumages de la semaine déclare: «Le rabbi disait que pour illuminer le monde il faut de la lumière... matérielle! Contre l'obscurité de la société actuelle, qui est bien éloignée de la loi de la morale». Une autre s'exclame: "La lumière de Hanoucca est là pour le monde, pas seulement pour les juifs» déclare à Paris une femme Loubavitch ${ }^{12}$. Ainsi les allumages publics des bougies de Hanoucca font partie de ces nouvelles «liturgies urbaines» (Abdullah, op. cit.) vécues comme des vecteurs de diffusion de la foi pour le monde urbain.

Les Loubavitch ne se contentent pas d'être présents et visibles dans l'espace public, mais vont chercher dans la rue parmi les passants ou parmi les participants à ces fêtes, les "juifs égarés » qu'ils souhaitent ramener au judaïsme. De jeunes hommes en costume noir et chapeautés sont chargés d'aller vers les passants et les participants de la fête pour leur demander s'ils sont juifs, et le cas échéant s'ils ont allumé les bougies de Hanoucca. Des hanoukkiot, les candélabres utilisés pour la fête de Hanoucca, des bougies ainsi qu'un livret explicatif de la fête et des coutumes sont gracieusement offerts.

En souhaitant placer une hanoukkia dans un maximum de maisons juives, les Loubavitch désirent rallumer la lumière symbolique de la spiritualité juive et réinscrire de la continuité identitaire après des ruptures de transmission des rites, fréquentes dans le contexte de sécularisation et dans le contexte des tragédies familiales et collectives vécues par les juifs au $\mathrm{xx}^{\mathrm{e}}$ siècle. Ces hanoukkiot gracieusement distribuées représentent un ancrage matériel du lien communautaire à partir duquel s'élabore, ou se reconstruit un sentiment d'appartenance: c'est justement pour « ressentir la communauté » dans la joie de la fête que des juifs non Loubavitch se rassemblent pour ces allumages collectifs.

10. Homme né en 1997, étudiant de yeshiva (Centre d'études talmudiques pour hommes mariés).

11. Commandements divins.

12. Femme d'une cinquantaine d'années, enseignante dans une école Loubavitch. 


\section{«Être avec la communauté »}

Même si l'origine de cette célébration publique est l'accomplissement d'un rite religieux et la volonté d'un mouvement messianique de ramener les juifs vers les mitsvot, tel n'est pas le sens donné à leur présence par tous les participants. En premier lieu, les personnes interrogées ont été nombreuses à mettre en avant explicitement la volonté d'appartenance au monde juif le temps d'une célébration, aisément désigné à Londres comme "une communauté » tandis que ce terme semble émerger moins fréquemment à Paris. Le mot communauté n'a pas la même résonance lorsqu'il est énoncé par le public dans le contexte français ou britannique. Dans le monde anglo-saxon ce mot est utilisé pour désigner toutes sortes de groupes sociaux, ainsi que des instances (supposées être) représentatives des faith communities (Beckford, opus cité). En France le mot «communauté » est considéré comme opposé à l'universalisme républicain et souvent associé au mot péjoratif «communautarisme ${ }^{13}$ ».

«La communauté juive » en tant qu'entité homogène, fermée, pérenne, n'existe que dans les représentations et les discours communs, politiques et journalistiques, alors que les sociologues montrent depuis longtemps la grande diversité du monde juif et les limites de la représentativité des institutions dites «communautaires ». Mais si l'image $d^{\prime}$ ' une » communauté unie émerge souvent au sein du public des allumages de Hanoucca, c'est qu'elle montre la capacité de l'événement à réactualiser un sentiment d'appartenance à un collectif: par exemple cet homme de 31 ans ${ }^{14}$ qui se définit comme pratiquant dit participer "pour être avec la communauté [...] C'est une manière de s'impliquer dans la fête ". Un homme de 72 ans ${ }^{15}$ qui se dit "légèrement pratiquant » déclare participer «pour apporter [son] soutien à la communauté juive londonienne». Si le terme «communauté» ne peut désigner l'ensemble de la population juive, la notion de communauté peut trouver une valeur heuristique lorsqu'elle désigne la représentation d'un collectif dont l'unité est imaginaire (Endelstein, 2008, 2016), et auquel la fête réactualise le sentiment d'appartenance. Ce sentiment d'appartenance s'exprime dans des liens sociaux qui se déploient concrètement dans la vie sociale, et de manière parfois événementielle dans l'espace public ${ }^{16}$.

Mais l'idée qu'il existe "une " communauté coexiste avec la conscience d'un rassemblement dans la diversité. Une femme ${ }^{17}$ "très pratiquante » d'une cinquantaine d'années, qui fréquente une synagogue orthodoxe déclare: «Tout le monde se rassemble. Des juifs et des personnes intéressées; des gens religieux

13. Pour une réflexion sur le débat social autour des communautés et minorités religieuses en France, voir Obadia et Zwilling (2016).

14. Homme né en 1984 , chercheur.

15. Homme né en 1944, ingénieur.

16. Sur l'articulation entre la matérialité des liens sociaux et la production d'un imaginaire communautaire, et pour une critique de la notion de «communautés imaginées» de Benedic Anderson, voir Chivallon (2007).

17. Femme d'une cinquantaine d'années, consultante technique. 
et des gens moins religieux, ils se rassemblent, c'est incroyable ». L'émotion produite par ces rassemblements ${ }^{18}$ est au cœur du mécanisme qui permet de dépasser la diversité des parcours, des sensibilités et affiliations religieuses au profit d'une identité juive commune ${ }^{19}$.

Il s'agit là sans doute d'un succès majeur des actions spectaculaires du mouvement Loubavitch: ses allumages publics sont quasiment les seules occasions susceptibles de rassembler, pour un temps limité, le spectre élargi du monde juif. Nous touchons ici à l'originalité du mouvement Loubavitch au sein du monde hassidique et à ses paradoxes: ultra-orthodoxe, il est capable de rassembler même temporairement le monde juif; prônant un «retour» à des pratiques strictes, il utilise les moyens les plus modernes de communication comme autant de vecteurs de diffusion locale et planétaire de ses messages. Faisant de ces paradoxes son originalité et sa force, ce mouvement suscite au sein du monde juif tantôt le rejet et la réprobation, tantôt l'admiration et la sympathie pour ses efforts constamment déployés pour rassembler et réanimer le monde juif.

\section{Appartenances événementielles}

Pour ceux qui se déclarent «non pratiquants » ou "légèrement pratiquants ", la dimension religieuse de l'événement et la croyance au miracle sont explicitement mises de côté, au profit de ses dimensions sociale et identitaire. Un jeune étudiant parisien, qui dit "faire les fêtes et c'est tout », autrement dit ne pas observer le shabbat et ne pas être pratiquant, déclare: "Je suis venu par tradition plus que par religion". À Londres, pour une femme ${ }^{20}$ «légèrement pratiquante ", "c'est un incontournable de la religion juive même si vous n'êtes pas orthodoxe». Ces discours et le sens donné à cette célébration collective par les juifs les moins pratiquants montrent bien comment une pratique religieuse peut être utilisée à des fins identitaires. Refusant les contraintes de l'observance maximaliste de la Loi juive, si omniprésente dans la vie quotidienne des ultra-orthodoxes, les participants juifs peu ou pas pratiquants viennent affirmer et ressentir leur appartenance au monde juif.

De nombreux juifs ont en effet recours à la religion pour fonder leur identité tout en ayant des relations irrégulières voire très distendues avec l'observance religieuse. Une grande partie d'entre eux de par le monde sélectionnent et accomplissent certaines pratiques dans le but de s'inscrire dans un collectif communément désigné comme «le peuple juif» : c'est ce qu'Herbert Gans a nommé une «religiosité symbolique»(Gans, 1994). Par la célébration des

18. Les rituels et l'émotion collective ont un impact profond sur le sentiment d'intégration dans un groupe. Voir Knottnerus (2014).

19. La fusion de la diversité dans une unité événementielle peut s'observer au cours d'autres rassemblements religieux, tels que les Journées Mondiales de la Jeunesse par exemple. Voir à ce sujet Jean-Philippe Perreault, "Le pluralisme uniformisant des JMJ », http://presence-info.ca/ article/opinion/le-pluralisme-uniformisant-des-jmj

20. Pas de réponse aux questions concernant l'âge et la profession. 
principales fêtes, en se rendant épisodiquement dans les lieux de culte (qui sont des lieux de rencontre et de sociabilité), en participant à des événements tels que l'allumage des bougies de Hanoucca, c'est l'appartenance à un collectif qu'ils expriment plutôt qu'une "tradition appréhendée comme principe normatif » impliquant un "ritualisme plus prononcé [...] au sens d'une conformité à une praxis » (Tank-Storper, 2015). C'est ce que signifie l'auto-désignation des juifs qui se déclarent «traditionnalistes ». Cette expression courante met en avant une interprétation de la tradition qui privilégie l'attachement à un héritage et sa transmission, plutôt que l'observance des commandements. S'il existe une transcendance dans l'accomplissement de ces rituels en public, celle-ci réside dans le sentiment d'appartenance à un peuple-monde transhistorique plutôt que dans un rapport au divin.

D'autres participants sont «juifs de loin» ou viennent pour affirmer leur solidarité en raison des liens familiaux qui les unissent au monde juif : à Golders Green, une femme ${ }^{21}$ hongroise, chrétienne, dit assister à l'allumage de Hanoucca "parce que j'aime le peuple juif. Dans ma famille j'ai une grand mère qui était juive». Un homme non juif ${ }^{22}$, qui ne pratique aucune religion, vient pour exprimer sa solidarité avec sa femme juive: "J'aime participer à ces événements de toutes façons, et c'est pour montrer ma solidarité avec ma compagne». Pour les conjoints non-juifs des mariages mixtes qui peinent à se faire reconnaître comme membres du monde juif lorsqu'ils le désirent, en particulier par les mouvements orthodoxes, le caractère public de ces événements festifs permet d'exprimer leur sympathie et de se sentir inclus dans ce collectif.

Enfin, c'est aussi pour raccommoder un lien relâché par l'histoire familiale ou la sécularisation, que certains viennent jusqu'à cet événement qui les rapproche pour quelques instants d'un monde juif qu'ils connaissent à peine. Ces «spectateurs de la frontière » du monde juif tendent à se positionner en retrait dans l'espace des festivités, là où se placent également les passants qui s'approchent par curiosité. Sur l'esplanade du Champ de Mars, une femme se tient à distance sur son vélo et observe la fête. Elle dit pourtant être venue exprès, même si elle ne sait pas expliquer le sens de la célébration. Elle dévoile sa judéité en déclarant: "Si on n'est pas juif on ne vient pas... », puis elle repart aussi furtivement qu'elle s'est approchée. À Trafalgar Square, un jeune lycéen de 17 ans dit qu'il participe à l'événement "pour en savoir plus sur le judaïsme ». Il a de lointaines origines juives, veut pratiquer et se rapprocher mais ne sait pas comment. Il s'agit précisément des personnes que les Loubavitch cherchent à toucher avec leurs actions de rue. Existe-t-il un lien entre cette participation et l'adoption durable d'une pratique du judaïsme? Même minoritaire, ce profil montre en tout cas que le mouvement Loubavitch atteint son objectif initial d'attirer des juifs isolés des réseaux de sociabilité juifs sur les lieux de célébrations.

21. Femme née en 1960, femme de ménage.

22. Homme né en 1946, retraité. 
Les fêtes de Hanoucca parviennent à rassembler dans la diversité, « du cœur aux confins des mondes juifs » (Bordes-Benayoun, 2013). Par-delà le monde juif, elles s'adressent aux spectateurs urbains, du passant au sympathisant.

\section{Faire la fête avec la société : regards croisés}

\section{Célébrer les racines juives du christianisme}

Dans les deux capitales, ces festivités attirent chaque soir le public peu nombreux mais fidèle d'un ensemble de sympathisants venus exprimer leur solidarité. Certains participants à la fête se sentent solidaires de ces célébrations pour des raisons religieuses: chrétiens très fervents, ils se sentent "juifs d'origine ". En célébrant les fêtes juives, ils viennent reconnaitre les racines juives du christianisme comme le déclare cette femme ${ }^{23}$ protestante évangélique: «Je participe à cet événement parce que je suis chrétienne. Mais le christianisme a des racines juives ». On rencontre aussi des «juifs pour Jésus ", mouvement qui reconnait Jésus comme le Messie des juifs et qui pratique conjointement des rites juifs et chrétiens, Jésus n'ayant pas eu, d'après eux, l'intention de fonder une nouvelle religion. À Trafalgar Square, une femme ${ }^{24}$ se déclare membre de ce mouvement de juifs messianiques, et explique : "[je participe] parce que je crois en Jésus mais tout vient du peuple juif, donc j’aime partager cela ». À Paris, deux femmes ${ }^{25}$ catholiques expliquent avoir pris le prospectus de Hanoucca distribué par les Loubavitch rue des Rosiers, et déclarent suivre les allumages chaque année. "On est catholiques mais on ne rejette pas l'Ancien Testament. On s'intéresse à la kabbale. À l'église pendant la messe on lit l'Ancien Testament. Et on va dans les synagogues au moment des grandes fêtes en Israël ». D'autres sympathisants chrétiens saisissent l'occasion de cette scène ouverte pour découvrir et célébrer une fête juive. Une femme ${ }^{26}$ accompagnée de sa fille se présente comme une protestante pentecôtiste: "J'ai beaucoup d'amis juifs, je veux seulement célébrer cette fête. Ma fille étudie le judaïsme à l'école ${ }^{27}$ ».

S'il ne s'agit pas de célébrations interreligieuses officielles et institutionnelles, qui se déroulent dans d'autres contextes publics et privés (Lamine, 2004), ces festivités religieuses en public offrent des occasions d'interaction aux frontières du monde juif, qui rendent possibles des démarches individuelles de connaissance du judaïsme et de reconnaissance de la différence religieuse. Des passants de hasard et des participants plus ou moins avertis font eux aussi partie de ce public hétéroclite.

23. Femme d'une cinquantaine d'années, puéricultrice.

24. Femme née en 1950, travailleuse social.

25. Femmes d'une cinquantaine d'années, professions non renseignées.

26. Femme née en 1958, consultante en recrutement.

27. Dans le contexte multiculturaliste du Royaume-Uni, les religions sont au programme des enseignements scolaires publics. 


\section{«Faire la fête avec les autres»}

Les allumages publics de Hanoucca mettent en scène la rencontre du monde juif avec la société urbaine, ce dont les participants semblent souvent conscients. À Paris comme à Londres, des enquêtés juifs déclarent explicitement vouloir convier la société à la fête, comme l'explique un couple parisien ${ }^{28}$ qui se déclare «traditionnaliste " : "Cette fête c'est le rassemblement, la communion, l'ouverture sur la ville et sur la cité; parce que tout le monde peut s'y joindre. C'est pour tout le monde. On essaie d'ouvrir la cité à cette fête».

Une femme retraitée qui se définit comme "assez pratiquante mais pas Loubavitch» considère que "c'est bien de faire la fête avec les autres». L'ouverture sur la ville est aussi mise en avant par des participants juifs londoniens. Un homme ${ }^{29}$ souligne ainsi «Tout le monde peut venir participer... On n'a pas besoin d'être juif. On veut tous que l'humanité soit une grande famille ". Une femme ${ }^{30}$ qui se définit très pratiquante, fréquentant une synagogue orthodoxe insiste: "C'est bien que des non-juifs voient cela». Ces allumages publics peuvent ainsi être vécus comme des occasions d'échange avec la société.

Dans le cas particulier du monde juif, inscrit dans une longue histoire de persécutions, et dans le contexte des violences des années 2000 et 2010 à l'encontre de personnes et de lieux juifs en Europe ${ }^{31}$, ces fêtes prennent aussi une dimension plus grave. C'est ce que dit le couple parisien précédemment cité, en 2011: «Et puis il y a un désir de continuer à exister. Après toutes les tentatives pour nous faire disparaître, c'est un témoignage qu'on est là, pour continuer à exister ». La sinistre réalité des attentats de novembre 2015 à Paris est venue renforcer cette démonstration, les allumages publics de Hanoucca ayant pris un sens de résistance à la menace physique et de continuité existentielle ${ }^{32}$. Un homme ${ }^{33}$ né en 1983, qui se dit «légèrement pratiquant» déclare: "Ça fait toujours plaisir de voir les allumages, surtout aujourd'hui quand les gens ont peur de montrer qu'ils sont juifs ». Une femme âgée de 85 ans $^{34}$, qui se définit juive libérale et légèrement pratiquante explique que cette fête symbolise les valeurs de liberté et de respect de son pays: «Cela veut dire la liberté, le respect des autres, mais nous pouvons nous exprimer. Nous vivons dans un pays dans

28. Couple, environ cinquante ans, directrice commerciale et agent immobilier.

29. Homme né en 1930, retraité.

30. Femme d'une cinquantaine d'années, consultante technique.

31. Voir par exemple pour la France les rapports de la Commission nationale consultative des droits de l'Homme (CNCDH):

http://www.cncdh.fr/fr/publications/rapport-sur-la-lutte-contre-le-racisme-lantisemitisme-et-laxenophobie-2014

32. À Paris les allumages publics de Hanoucca ont failli être tous annulés par la Préfecture, de nombreux allumages ont finalement été maintenus dont celui du Trocadéro en duplex avec Jérusalem et New York.

33. Homme né en 1983, informaticien.

34. Femme née en 1930, retraitée. 
lequel nous pouvons exprimer le vrai sens de notre croyance. Cela signifie le respect pour les autres, la sécurité pour tous. Pas de radicalisme». Cette dernière affirmation peut sembler étonnante pour désigner une fête organisée par un mouvement juif ultra-orthodoxe, qui tente de propager l'application de la loi juive et qui se tourne vers l'observance extrême des mitsvot. C'est justement le propre de la stratégie du mouvement Loubavitch qui ne cherche pas à imposer un mode de vie religieux et se contente de «donner l'exemple» en rendant publics des rites d'une manière festive et joyeuse.

Ce caractère très festif des allumages de Hanoucca les rend potentiellement attractifs pour un plus large public, notamment sur les lieux touristiques. À Londres, deux jeunes femmes ${ }^{35}$ de 26 et 36 ans, l'une "sans religion » et l'autre "culturellement musulmane ", se sont donné rendez-vous à l'allumage de Trafalgar Square pour se livrer à une activité gratuite au moment où elles souhaitaient se retrouver: "Nous voulions nous rencontrer jeudi et faire quelque chose gratuitement ». L'événement figurait sur le site web de la Ville de Londres, ainsi que parmi les «les choses gratuites à faire à Londres» du Time Out. Aux yeux de ce public, la fête de Hanoucca est une fête urbaine comme les autres, dont le sens religieux est à peine saisi, permettant de se rassembler avec un public joyeux, d'écouter de la musique et de bénéficier d'une distribution gratuite de beignets. Les Loubavitch en barbe, chapeau et costume sombre sont simplement considérés comme de curieux protagonistes de l'événement... La présence de touristes sur les places majeures de ces célébrations indique que les lieux choisis et négociés jouent un rôle décisif dans les échanges qu'ils permettent entre le monde juif et l'ensemble du monde urbain.

\section{Symbolique des lieux}

L'importance des lieux où se déroulent les fêtes, processions, manifestations de rue a déjà été fréquemment soulignée. Les significations politiques des modes d'investissement de l'espace ont retenu l'attention d'historiens des marches et manifestations (Pigenet et Tartakowsky, 2003) tandis que dans les approches géographiques des fêtes, les lieux ne sont pas considérés comme "de vulgaires cadres de l'action festive", mais comme "des acteurs signifiants de l'événement [...], consubstantiels de la fête» (Di Méo, 2001). Dans le cas présent les lieux participent ainsi, autant que les usages, à la ritualisation de la rencontre entre le monde juif et la société.

Dans les deux capitales, les lieux choisis pour le déroulement de certaines festivités sont chargés d'une grande puissance symbolique puisqu'il s'agit de hauts lieux des nations française et britannique: le Champ de Mars devant la Tour Eiffel, l'Arc de Triomphe, la place de la République, Trafalgar Square, etc. Ces contextes spatiaux interviennent dans la fête de manière déterminante, en superposant pour une durée très limitée les symboles du monde juif et ceux de

35. Femmes nées en 1979 et 1989, profession non déclarée. 


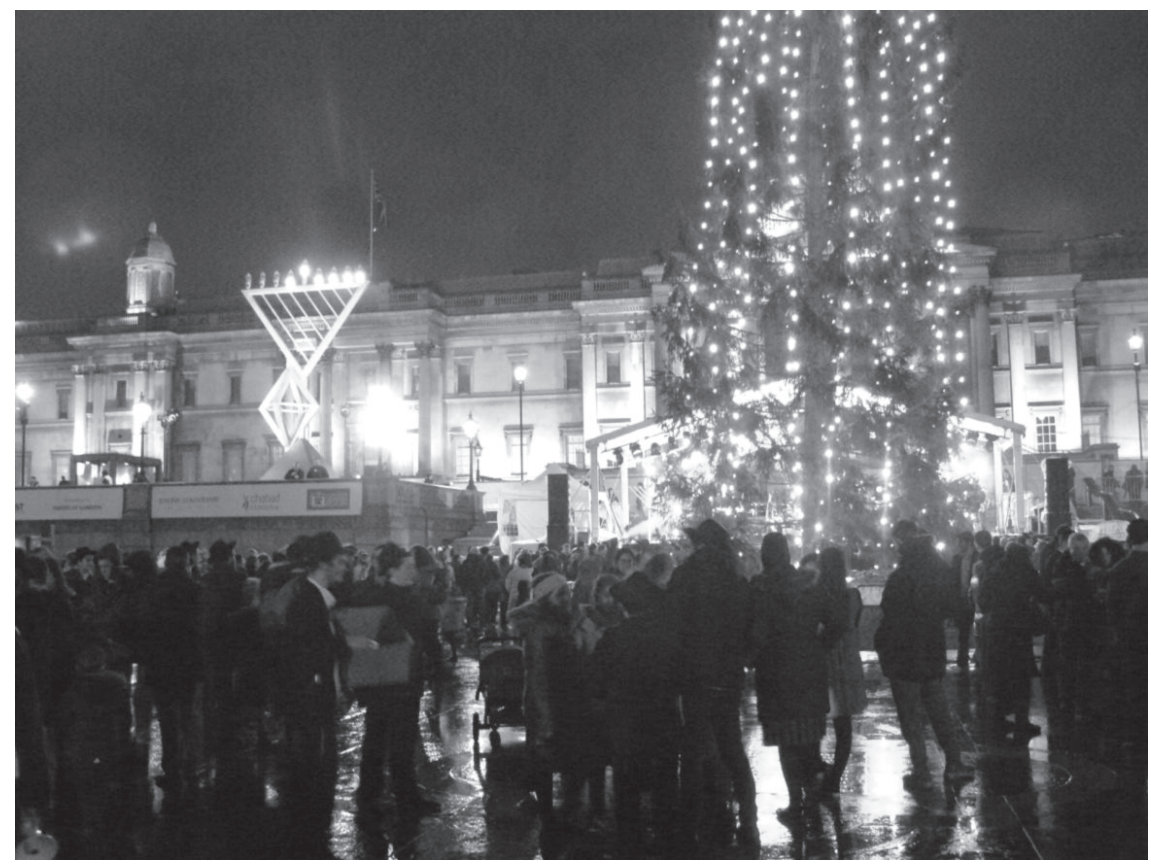

Photo 3. Allumage des bougies de Hanoucca à Trafalgar Square, Londres, décembre 2015, photo de l'auteur

la nation d'installation, produisant ainsi des images insolites de candélabres géants en arrière-plan desquels figurent les principaux monuments des capitales (photo 3). Ces images sont ensuite pérennisées sur les différents médias utilisés par le mouvement Loubavitch: sites internet, prospectus d'information, affiches, etc. La nation n'est pas présente uniquement dans la symbolique des lieux, mais également dans l'action festive: les orchestres jouent souvent les hymnes nationaux, et les autorités publiques sont systématiquement remerciées par les responsables communautaires pour leur autorisation à tenir ces fêtes, en affirmant ainsi leur lien au politique et la légitimité de la place d'une fête juive dans les espaces publics.

Quels sont les enjeux durables de ces images et de ces actions superposant les références à la Nation et les références au monde juif? Au-delà de la recherche de légitimité précédemment évoquée autour de la négociation de lieux avec les autorités locales, il existe pour le monde Loubavitch une volonté manifeste d'être présent au cœur des nations. En effet cette spectacularisation du judaïsme sur les lieux les plus symboliques des capitales met en scène l'idéologie politico-religieuse du mouvement Loubavitch, pour lequel la présence de ses émissaires dans toute la diaspora est fondamentale pour ramener les juifs de par le monde aux pratiques des mitsvot et hâter la venue du Messie. C'est pourquoi ce mouvement ne prône pas l'Alya - immigration des juifs en Israël - et se distingue des autres mouvements hassidiques qui 
ne recherchent pas les contacts avec le reste du monde juif. Les Loubavitch accordent à leur place dans chaque nation une importance capitale, puisque c'est en faisant pleinement partie de la société qu'ils peuvent y diffuser leurs messages et remplir leur mission de prosélytisme au sein du monde juif.

Par ailleurs pour le public élargi, ces fêtes ne sont-elles pas une occasion de prendre place en tant que juif dans la société et de devenir visibles le temps d'une cérémonie ? Si la fête, comme le pèlerinage, est un moment privilégié de cristallisation du sentiment d'appartenance à une communauté, n'est-elle pas un moment où s'expose aussi l'appartenance à une nation ? C'est ce que semble indiquer l'auto-identification des participants interrogés, qui se définissent presque systématiquement par leur judéité et par leur nationalité. En considérant la fête avec Guy Di Méo comme «l'expression territoriale, à la fois concrète et symbolique, d'une collectivité sociale cherchant à affirmer son identité en se servant des lieux à cette fin » (2001: 643), la combinaison dans l'espace des symboles du judaïsme et de la nation peut être regardée comme une manière de «prendre place» en tant que juifs français ou britanniques dans la cité.

$$
* *
$$

Les allumages publics des bougies de Hanoucca jouent un rôle ambivalent à l'égard de l'altérité: festivités ouvertes sur la ville, lieux d'échanges et de liesse partagée, elles cultivent en même temps le particularisme de l'appartenance au monde juif et les normes strictes d'un mouvement ultra-orthodoxe. Ces fêtes urbaines sont emblématiques des paradoxes de la transnationalisation religieuse qui «confronte le chercheur au défi de comprendre comment se met en place cette dynamique qui conjugue cosmopolitisme et particularisme, fermeture communautaire et ouverture sur le monde». (Capone, 2010: 247). Porteuses d'une attente messianique aussi bien que de démarches identitaires pouvant être dissociées du croire, ces célébrations participent aussi au niveau collectif d'une recherche de reconnaissance au sein des nations d'installation.

Donner la parole au public des allumages de Hanoucca organisés par le mouvement Loubavitch a mis en lumière des expériences multiples de la fête et a donné à voir le monde juif mis en spectacle comme un collectif hétérogène et aux frontières externes floues. La fête offre une occasion pour voir se brouiller - ne serait-ce que temporairement - les frontières entre la religion institutionnalisée et non institutionnalisée, entre le monde de l'ultra-orthodoxie et le monde urbain, entre les différents courants du judaïsme. L'émotion du rassemblement ravive le sentiment d'appartenance et résout la tension entre la diversité du public et l'idée qu'il existe une "communauté » unie, locale et diasporique - bien que cette fête n'existerait pas sans la volonté des Loubavitch d'attirer des juifs vers la pratique religieuse.

Ces rencontres festives ouvrent ainsi des questions sur les images du monde juif produites et éventuellement pérennisées par ces événements, qui nécessitent d'être étayées par des enquêtes ultérieures. Avec la mise en scène de l'ultra-orthodoxie comme organisatrice des festivités, c'est une lecture religieuse 
du monde juif qui est proposée pendant ces fêtes et l'on peut supposer que ces dernières contribuent, avec d'autres événements, à renforcer l'image du monde juif comme étant une minorité religieuse (Cohen, 2014), bien que la judéité dépasse l'identification à une religion. Alors que la diversité du public juif s'expose pendant ces fêtes, celle-ci échappe à l'observation des passants non avertis. Ces festivités peuvent-elles alors contribuer à (re)construire l'imaginaire d'une «communauté juive » homogène et solidaire ? Tendent-elles par ailleurs à banaliser la présence juive dans la cité ? La dimension religieuse de ces célébrations publiques contribue-t-elle à reconstruire l'image d'une différence qui serait éternellement associée au monde juif?

On peut enfin interroger le rôle du statut public accordé à la religion selon chaque contexte national dans les perceptions de ces fêtes urbaines par les citadins. Le Royaume-Uni accordant plus de place aux religions dans la vie publique et quotidienne, alors que la visibilité religieuse fait l'objet de virulents débats en France, la mise en spectacle du judaïsme est-elle reçue différemment dans les deux pays? Le caractère festif et extraordinaire de ces allumages publics les renvoie à un folklore urbain dont l'existence est peut-être moins polémique que la visibilité religieuse ordinaire et les prières de rue.

\author{
Lucine ENDELSTEIN \\ CNRS LISST-CIEU (UMR 5193) \\ Université Toulouse Jean Jaurès \\ lucine.endelstein@univ-tlse2.fr
}

\title{
Bibliographie
}

Abdullah Zain, 2009, "Sufis on parade: the performance of Black, African, and Muslim identities", Journal Of The American Academy Of Religion. American Academy Of Religion, vol. 77, n 2, p. 199-237.

Baby-Collin Virginie, Sassone Susana, 2010, «Mondialisation de la Virgen de Urkupiña ? Religiosité, fêtes populaires et territoires urbains des migrants boliviens, de Buenos Aires à Madrid.", Autrepart, no 56, p. 111-132.

BAUMgARTEN Jean, 2005, La naissance du hassidisme : mystique, rituel, société (XVIII ${ }^{e}$ XIX ${ }^{e}$ siècle), Paris, Albin Michel.

BeCKFord J. A., 2015, " "Community” in the Sociology of Religion: The Case of Britain ", Social Compass, vol. 62, n 2, p. 225-237.

Bordes-Benayoun Chantal, 2013, «Du cœur aux confins de la judéité », Ethnologie française, vol. 43, no 4, p. 573-579.

Campbell Heidi A., éd. 2013, Digital Religion: Understanding Religious Practice in New Media Worlds, Abingdon, Oxon, New York, Routledge.

Capone Stefania, 2010, "Religions “en migration": de l'étude des migrations internationales à l'approche transnationale ", Autrepart, no 56, p. 235-259.

Chivallon Christine, 2007, «Retour sur la “communauté imaginée” d'Anderson.» Raisons politiques, vol. 27, $\mathrm{n}^{\circ}$ 3, p. 131-172. 
Cohen Martine, 2014, «Les juifs de France sont-ils en train de redevenir une minorité religieuse? ", Obadia L., Zwilling A.-L. (éds.), Minorité et communauté en religion, Strasbourg, Presses universitaires de Strasbourg, p. 151-166.

Di Méo Guy, 2001, «Le sens géographique des fêtes » [The geographical meaning of festivities], Annales de Géographie, vol. 110, nº 622, p. 624-646.

Di Méo Guy, 2005, «Le renouvellement des fêtes et des festivals, ses implications géographiques ", Annales de géographie, no 643, p. 227-243.

Duteil-Otaga Fabienne, Jonveaux Isabelle, Kuczynski Liliane, Nizard Sophie (éds.), 2015, Le religieux sur internet, Paris, L'Harmattan.

Eade John, Garbin David, 2007, "Reinterpreting the Relationship Between Centre and Periphery: Pilgrimage and Sacred Spatialisation Among Polish and Congolese Communities in Britain”, Mobilities, vol. 2, no 3, p. 413-424.

ENDELSTEIN Lucine, 2008, Une géographie du renouveau religieux. Judaïsme et expérience urbaine en quartier cosmopolite. Paris, $19^{e}$ arrondissement, Université de Poitiers, Thèse de doctorat.

Endelstein Lucine, 20I6, «Religion et communautés juives. Pour une approche spatiale de la diversité communautaire ", L’Information Géographique, no 1, p. 14-21.

Fath Sébastien, 2010, "Fondamentalisme », in Azria R., Hervieu-Léger D. (éds.), Dictionnaire des faits religieux, Paris, Presses universitaires de France.

Fer Yannick, 2007, «Pentecôtisme et modernité urbaine: entre déterritorialisation des identités et réinvestissement symbolique de l'espace urbain ", Social Compass, vol. 54, $\mathrm{n}^{\circ} 2$, p. 201-210.

Gans Herbert J., 1994, “Symbolic Ethnicity and Symbolic Religiosity: Towards a Comparison of Ethnic and Religious Acculturation”, Ethnic and Racial Studies, vol. 17, $\mathrm{n}^{\circ} 4$, p. 577-592.

GARbin David, 2012a, "Introduction: Believing in the City ", Culture and Religion, vol. $13, \mathrm{n}^{\circ} 4$, p. $401-404$.

-, 2012b, «Marching for God in the Global City: Public Space, Religion and Diasporic Identities in a Transnational African Church ", Culture and Religion, vol. 13, $\mathrm{n}^{\circ} 4$, p. 425-447.

GuTwIRTH Jacques, 2004, La renaissance du hassidisme: de 1945 à nos jours, Paris, Odile Jacob.

Jarrassé Dominique, 2001, Synagogues : une architecture de l'identité juive, Paris, Adam Biro.

Joseph Isaac (éd.), 1995, Prendre place: espace public et culture dramatique, Paris, Éditions Recherches Plan urbain.

Kershen Anne, Vaughan Laura, 2013, «"There was a Priest, a Rabbi and an Imam...”: an Analysis of Urban Space and Religious Practice in London's East End, 1685-2010 », Material Religion, vol. 9, n 1, p. 10-35.

KnotTnerus J. David, 2014, Religion, Ritual, and Collective Emotion, Oxford, Oxford University Press.

Kravel-Tovi Michal, 2009, «To see the Invisible Messiah: Messianic Socialization in the Wake of a Failed Prophecy in Chabad", Religion, vol. 39, n 3, p. 248-260.

LAmine Anne-Sophie, 2004, La cohabitation des dieux : pluralité religieuse et laïcité, Paris, Presses universitaires de France. 
LevitT Peggy, 2007, God Needs No Passport: Immigrants and the Changing American Religious Landscape, New York, London, New Press.

Obadia Lionel, Zwilling Anne-Laure (éds.), 2016, Minorité et communauté en religion, Strasbourg, Presses universitaires de Strasbourg.

Orsi Robert Anthony, éd. 1999, Gods of the City : Religion and the American Urban Landscape, coll. Religion in North America, Bloomington, Indiana University Press.

PAquot Thierry, 2015, L’Espace public, Paris, La Découverte.

Pigenet Michel, TARTAKowsky Danielle, 2003, «Les territoires des mouvements sociaux », Le Mouvement Social, vol. 202, n 1, p. 3-13.

Podselver Laurence, 2010, Retour au judaïsme ? Les Loubavitch en France, Paris, Odile Jacob.

SAlzbrunn Monika, 2014, "Appartenances en fête: entre l'ordinaire et le spectaculaire ", Social Compass, vol. 61, $\mathrm{n}^{\circ}$ 2, p. 250-260.

TANK-Storper Sébastien, 2015, «Orthodoxies en concurrence. Essai sur la pluralité socio-religieuse dans le judaïsme contemporain », Bordes-Benayoun C. (éd.), Socioanthropologie des judaïsmes contemporains, Paris, Honoré Champion, p. 65-78.

\section{Lumières sur la ville. Les fêtes de Hanoucca entre action missionnaire transnationale et appartenance événementielle}

Les fêtes de Hanoucca sont organisées par le mouvement Loubavitch depuis les années 1980 dans les espaces publics des métropoles du monde entier. Elles relèvent d'une action missionnaire qui vise à ramener un maximum de juifs à la pratique du judaïsme. Des enquêtes auprès des publics à Paris et à Londres révèlent que ces festivités organisées par un mouvement ultraorthodoxe parviennent à rassembler le monde juif dans sa diversité ainsi que des sympathisants non juifs. En brouillant temporairement les frontières entre l'ultra-orthodoxie et le monde urbain et entre les différents courants $d u$ judaïsme, ces événements permettent d'analyser le rôle de la religion sous sa forme transnationale et événementielle dans la (re)construction du sentiment d'appartenance au monde juif, et dans la négociation d'une place dans la ville.

Mots-clés: événement, pratiques religieuses transnationales, espaces publics, appartenance, judaïsme.

\section{Lights in the city. The festival of Hanukka between transnational missionary action and event belonging}

Since the 1980's the festival of Hanukka has been organised by the HabadLubavitch movement in the public spaces of the metropolis around the world. They are part of a missionary action that aims to bring as many Jews as possible to the practice of judaism. Surveys among the publics in Paris and London reveal that these festivals organized by an ultra-orthodox movement bring together the Jewish world beyond its own diversity along with non-Jewish sympathisers. Those events participate in a temporay bluring of boundaries 
between the urban and the ultra-orthodox worlds, and between the different movements within Judaism. They provide an opportunity to examine the role of transnational religious events in the (re)building of the belonging to the Jewish world and in negotiating a place in the city.

Keywords: festivals, transnational religious practices, public space, belonging, judaism.

\section{Luces sobre la ciudad. Las fiestas de Hanuka entre ación misionaira transnacional y pertenencia de eventos}

Desde los años 1980 las fiestas de Januca están organizadas por el movimiento Lubavitch en las metropolis del mundo. Forman parte de una accione misionaria cuyo ámbito es traer al máximo de judios a la practica del judaísmo. Unas encuestas sobre los publicos en Paris y en Londres revelan que estas fiestas organizadas por un movimiento ultra-orthodoxo consiguen reunir al mundo judio en toda su diversidad, asi que simpatizantes no judios. Estas celebraciones desdibujan temporalmente les fronteras entre el mundo ultraortodoxo y el mundo urbano y entre los diferentes movimientos del judaísmo. Permiten analizar el papel de acontecimientos religiosos transnacionales en la (re)construcción de la pertenencia al mundo judio, y en la negociación de una plaza en la ciudad

Palabras clave: acontecimientos, practicas religiosas transnacionales, espacio publico, pertenencia, judaísmo. 